\title{
Selective Intra-Arterial Embolization for Advanced Extrascleral Uveal Melanoma
}

\author{
Víctor M. Villegas ${ }^{a, b}$ Mariola Monagas ${ }^{a}$ Joseph Campbell ${ }^{a}$ \\ Timothy G. Murray ${ }^{c}$ Luis Serrano ${ }^{a}$ \\ a Department of Ophthalmology, University of Puerto Rico, Río Piedras, Puerto Rico; b Bascom Palmer Eye Institute, \\ University of Miami, and 'Murray Ocular Oncology and Retina, Miami, FL, USA
}

\section{Keywords}

Melanoma $\cdot$ Malignant melanoma $\cdot$ Ocular melanoma

\begin{abstract}
Purpose: To report a treatment approach for advanced extrascleral uveal melanoma. Methods: We performed clinical examination including magnetic resonance imaging, computed tomography, angiography, and histopathologic analysis. Case: A 49-year-old healthy woman presented with a 7-year history of an enlarging pigmented mass in her right orbit. Malignant melanoma was diagnosed after biopsy with immunohistochemical stains. Treatment included selective intra-arterial embolization. Results: A significant reduction in tumor burden was seen 3 months after intra-arterial embolization. No complications were associated with the treatment. Conclusion: Selective intra-arterial embolization may allow adequate palliative therapy in select cases of advanced extrascleral uveal melanoma.

(c) 2017 S. Karger AG, Basel
\end{abstract}

\section{Introduction}

Uveal melanoma is the most common primary intraocular malignancy in adults [1]. The two most important clinical variables associated with survival are size and cell

\section{KARGER}

(C) 2017 S. Karger AG, Basel

E-Mail karger@karger.com

www.karger.com/oop type [2]. Metastasis occurs in up to $50 \%$ of cases and the most common site is the liver [1-3]. Small and mediumsized uveal melanomas can be treated with globe-sparing treatments. However, the mainstay of treatment of large choroidal melanoma is enucleation.

Extrascleral extension of uveal melanoma is exceedingly rare and the treatment varies depending on the extent of the disease. Minimal extrascleral extension can be treated by brachytherapy [4]. Moderate extrascleral extension can be treated with enucleation and focal excision of orbital disease. This approach has historically been combined with external beam radiation to treat residual microscopic disease. Extensive extrascleral extension can be treated with exenteration and external beam radiation [5-8]. A recent report described brachytherapy as an alternative to external beam radiation treatment for postenucleation orbital melanoma [9].

In this report we describe the features present in an untreated patient with extensive extrascleral uveal melanoma, and we report the radiological imaging findings and novel therapeutic interventions performed to manage such a complex case.

This work was presented at the 2017 International Society of Ocular Oncology Biennial Conference in Sydney, Australia.

Víctor M. Villegas, MD

Bascom Palmer Eye Institute

900 NW 17th Street

Miami, FL 33136 (USA)

E-Mail v.villegas@med.miami.edu 


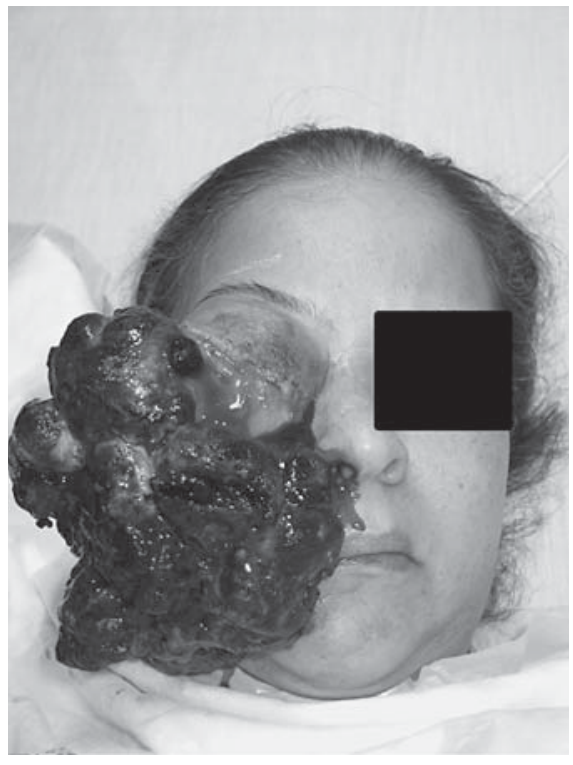

Fig. 1. Frontal view of the massive exophytic multilobulated pigmented tumor pushing against the lateral lip and nose of the patient.
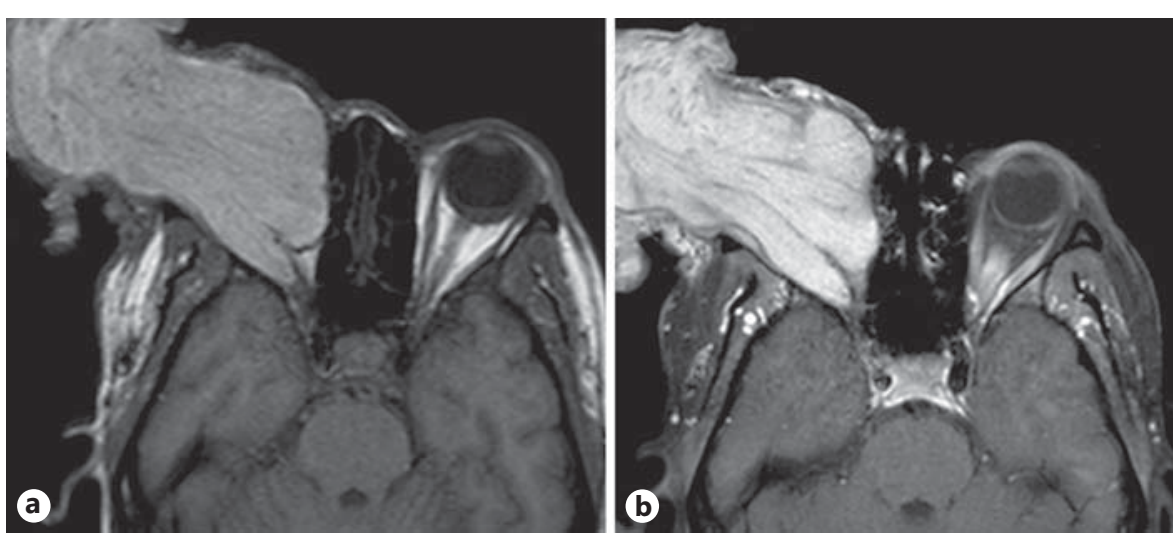

Fig. 2. Axial T1-weighted magnetic resonance imaging pre- (a) and post-contrast (b) images showed an avidly enhancing heterogeneous exophytic mass arising from the right orbit. It occupied the intra- and extraconal spaces, replacing the extraocular muscles, eye globe, and optic nerve. No extension into the cavernous sinus or other intracranial compartments was present.

\section{Case Report}

A 49-year-old woman without previous systemic medical history or allergies was evaluated at the emergency room of the University District Hospital Medical Center, Río Piedras, Puerto Rico due to progressive generalized weakness of 2 weeks' duration. Her past medical history included a slowly enlarging ocular mass that had been present for 7 years. At presentation, she complained of difficulty eating, drinking, and breathing due to mass compression of the facial structures and the associated constant putrid odor. The patient stated that the first sign of the mass had been a dark spot on the iris of her right eye. Five years prior she had developed sudden loss of vision. She denied foreign body sensation, pain, or visual symptoms when first noticing the lesion. As the tumor replaced the ocular tissues, she complained of foreign body sensation, inability to close the eyelids, spontaneous tumor necrosis, and bleeding. Pain was not reported at any stage. She also denied seeking prior medical evaluation due to religious ideation.

A complete ophthalmologic examination was performed. Bestcorrected visual acuity was 20/30 PH 20/20 in her left eye using the Snellen chart. Intraocular pressure as measured by applanation tonometry was $15 \mathrm{~mm} \mathrm{Hg}$ in her left eye. The right orbit had an exophytic, multilobulated, pigmented, putrid mass with associated necrotic tissue (Fig. 1). No globe structures were present. Examination of the contralateral eye was then performed. The cornea was clear. The anterior chamber was deep and quiet. The pupil was round and reactive to light. The lens was clear. Dilated fundus examination showed a clear vitreous, pink optic nerve with a 0.2 cup, intact foveal contour, vessels of normal size and pattern, and unremarkable retinal periphery.

Intra-Arterial Embolization for Uveal Melanoma
Laboratory workup showed a hemoglobin level of $3 \mathrm{~g} / \mathrm{dL}$ consistent with microcytic hypochromic anemia. The patient underwent multiple packed red blood cell transfusions until hemoglobin was $12 \mathrm{~g} / \mathrm{dL}$.

A magnetic resonance imaging study of the brain, orbits, and neck with and without gadolinium showed a large exophytic mass arising from the right orbit. It demonstrated the mass occupying the intra- and extraconal spaces with replacement of the extraocular muscles, eye globe, and optic nerve, without gross intracranial extension (Fig. 2). Abdominopelvic computed tomography revealed multiple intrahepatic masses. Thoracic computed tomography was remarkable for a left upper lobe pulmonary nodule with associated bilateral pleural effusions.

Incisional biopsy of the mass was performed. Microscopic examination revealed a neoplastic lesion composed of sheets of pigmented cells with irregular vesicular nuclei of various sizes and shapes, and dilated blood vessels with areas of hemorrhage. Immunohistochemical stains were positive for HMB-45 and Melan A with high Ki67, but negative for S100. The endothelial cells were positive for CD34 and CD31. Immunostains confirmed the diagnosis of malignant melanoma of the orbit.

The patient was then educated about her poor prognosis. A significant discussion was undertaken explaining the different palliative strategies. She elected to try to reduce the orbital tumor burden to improve her quality of life. Selective intra-arterial embolization was considered prior to exenteration to minimize intra- and postoperative hemorrhage. Partial excision of tumor was not considered because of the potential for poor hemostasis. Digital subtraction angiography showed hypervascularity mainly supplied by branches of the internal (Fig. 3) and external carotid arteries. The 

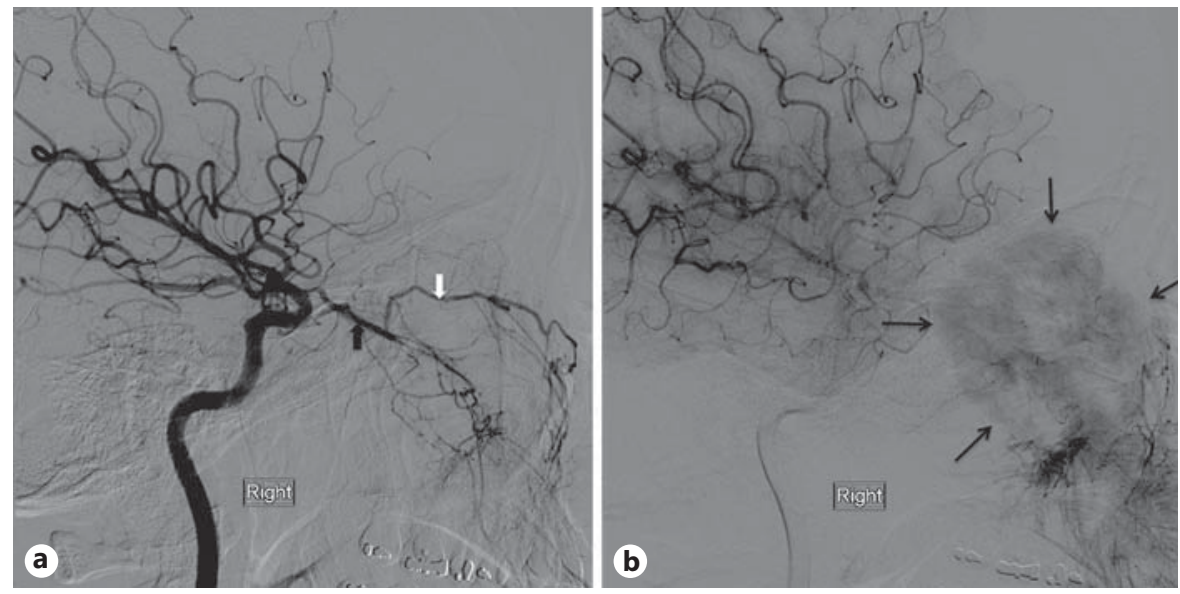

Fig. 3. Lateral views of digital subtraction angiography showing a large branching ophthalmic artery (black arrow) supplying the orbital and superior portion of the extraorbital tumor via the supraorbital artery (white arrow) (a). Late blush confirmed hypervascularity and location of tumor (multiple black arrows) (b).

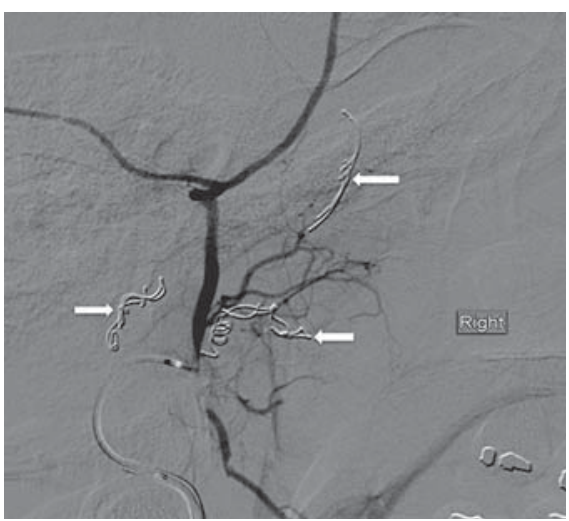

Fig. 4. Lateral views of digital subtraction angiography after coil embolization (white arrows) showing a marked decrease in flow to the mass without any evidence of late blush. ophthalmic artery was the only branch involved from the internal carotid artery. Branches from the internal and external carotid artery were selectively embolized using coils, with an approximately $70 \%$ reduction in blood flow (Fig. 4).

One day after embolization, exenteration was attempted but aborted due to insufficient hemostasis during the initial supraorbital incision. The patient was then discharged home with palliative care.

Three months after discharge the patient returned to the clinic for evaluation. Significant reduction in tumor burden was present (Fig. 5). She reported improved quality of life and did not want further intervention. Nine months after initial presentation, the patient died of complications arising from metastatic hepatic disease.

\section{Discussion}

Extraocular extension of uveal melanoma continues to represent a therapeutic challenge. The prognosis is poor once extraocular extension is present. Due to the rarity of extensive extraocular extension in uveal melanoma and limited reports, there is lack of consensus as to the best management strategy. The most common treatment options include isolated resection and modified enucleation or exenteration combined with radiotherapy or other forms of adjuvant therapy [5-13].

Uveal melanomas arising from the iris tend to follow a nonaggressive clinical course compared to choroidal melanomas [11]. Iris melanomas are usually deeply vascularized tumors that may cause spontaneous hyphema; however, metastases are rare in iris melanomas. The ori-

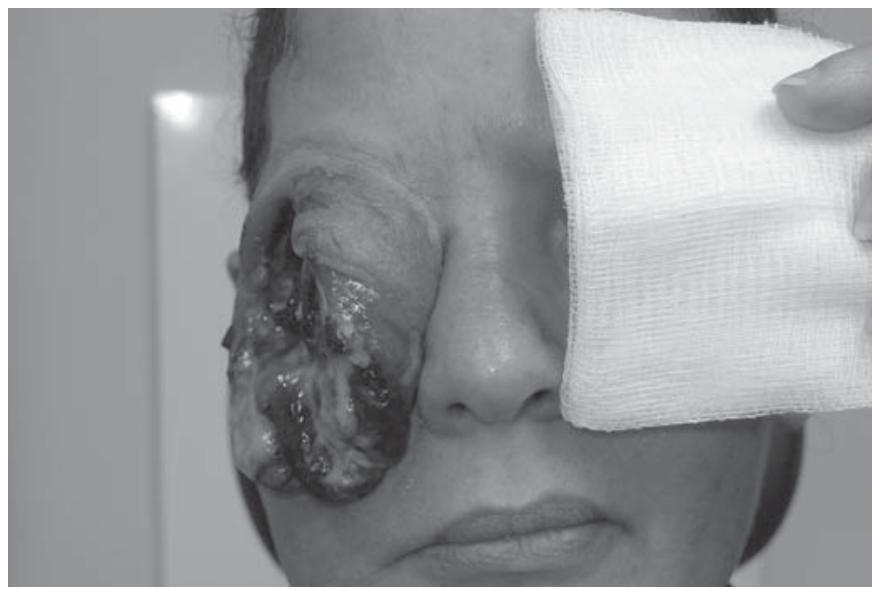

Fig. 5. Frontal view of the tumor 3 months after selective intraarterial embolization showing a significant decrease in tumor burden.

gin of the tumor in this case is unclear. It is unlikely to have originated from the iris. The tumor may have originated from a posterior uveal melanoma that may have extended through the iris.

Massive orbital extension of posterior uveal melanoma is exceedingly rare and is usually seen in patients who refuse treatment [12]. Therapy is typically palliative, with exenteration being the main option to decrease the tumor burden and improve the quality of life [13]. In this case, the abundant vascular supply of the tumor combined with the chronic anemia carried a significant mortality 
risk at the time of resection. Therefore, selective intraarterial embolization was attempted to decrease the vascular supply prior to exenteration. However, embolization did not preclude significant bleeding during surgery that impeded safe completion of the exenteration. This case is unique because selective intra-arterial embolization of the vascular supply of the tumor allowed significant reduction of the tumor burden without exenteration 3 months after treatment. As seen in our case, embolization of extensive orbital melanoma may provide adequate palliative care to some patients. Future studies are needed to better understand whether embolization can be successfully used in combination with other forms of treatment.

External beam radiation may be considered preoperatively to decrease tumor burden [13]. Post-exenteration external radiotherapy $(3,000-4,000 \mathrm{cGy})$ can be considered in cases who have not received previous radiotherapy. However, in cases with massive orbital extension, the risk of preoperative radiation may be higher than that of surgi- cal resection or embolization. Post-exenteration external radiotherapy may carry less morbidity in such cases.

The prognosis at the time of diagnosis remains an important topic. Patients with uveal melanoma and metastasis should be educated about the poor prognosis. Selective intra-arterial embolization may provide adequate palliative care in select patients with advanced extrascleral uveal melanoma.

\section{Statement of Ethics}

The subject in this paper gave informed consent prior to death. The institute's committee on human research has approved the manuscript.

\section{Disclosure Statement}

The submission did not receive any sponsorship or funding. No author in this paper has any possible conflicts of interest.

\section{References}

1 Shields JA, Shields CL, Donoso LA: Management of posterior uveal melanoma. Surv Ophthalmol 1991;36:161-195.

2 Shields CL, Kaliki S, Furuta M, Mashayekhi A, Shields JA: Clinical spectrum and prognosis of uveal melanoma based on age at presentation in 8,033 cases. Retina 2012;32:13631372.

3 Wykoff CC, Murray TG, Markoe AM, Feun LG, Schefler AC, Gayer S: Preoperative evaluation for patients with choroidal/ciliary body melanomas: what is necessary? Am J Ophthalmol 2011;151:921-924.

4 Augsburger JJ, Schneider S, Narayana A, Breneman JC, Aron BS, Barrett WL, Trichopoulos N: Plaque radiotherapy for choroidal and ciliochoroidal melanomas with limited nodular extrascleral extension. Can J Ophthalmol 2004;39:380-387.
5 Justusová P, Štubňa M, Veselovský M, Lipková B: Orbital exenteration in patient with metastatic choroidal melanoma - a case report (in Czech). Cesk Slov Oftalmol 2016;72: 92-96.

6 Chen C, Jamal KN, Hogan RN, Anand R: Aggressive amelanotic choroidal melanoma with extrascleral extension after brachytherapy. Retin Cases Brief Rep 2011;5:37-41.

7 Rahman I, Maino A, Cook AE, Leatherbarrow B: Mortality following exenteration for malignant tumours of the orbit. Br J Ophthalmol 2005;89:1445-1448.

8 Rosenberg C, Finger PT: Cutaneous malignant melanoma metastatic to the eye, lids, and orbit. Surv Ophthalmol 2008;53:187-202.

9 Finger PT, Tena LB, Semenova E, Aridgides P, Choi WH: Extrascleral extension of choroidal melanoma: post-enucleation high-doserate interstitial brachytherapy of the orbit. Brachytherapy 2014;13:275-280.
10 Blanco G: Diagnosis and treatment of orbital invasion in uveal melanoma. Can J Ophthalmol 2004;39:388-396.

11 Khan S, Finger PT, Yu GP, Razzaq L, Jager MJ, de Keizer RJ, Sandkull P, Seregard S, Gologorsky D, Schefler AC, Murray TG, Kivelä T, Giuliari GP, McGowan H, Simpson ER, Corriveau C, Coupland SE, Damato BE: Clinical and pathologic characteristics of biopsyproven iris melanoma: a multicenter international study. Arch Ophthalmol 2012;130:5764.

12 Shields JA, Shields CL: Massive orbital extension of posterior uveal melanoma. Ophthal Plast Reconstr Surg 1991;7:238-251.

13 Shields JA, Augsburger JJ, Corwin S, Donoso LA, Disantis M: The management of uveal melanomas with extrascleral extension. Orbit 1986;6:31-37. 